\title{
Computation of a cardiac severity score with left-censored biomarkers for patients with heart failure
}

This article was published in the following Dove Press journal:

Pathology and Laboratory Medicine International

14 February 2017

Number of times this article has been viewed

\author{
Anupama Vasudevan ${ }^{1,2}$ \\ Alyssa G Munkres ${ }^{3}$ \\ Teodoro Bottiglieri ${ }^{1,4}$ \\ Jane I Won ${ }^{1,2}$ \\ Puja Garg ${ }^{1,2}$ \\ Peter A McCullough ${ }^{1-3,5,6}$ \\ 'Baylor Research Institute, ${ }^{2}$ Baylor \\ Heart and Vascular Institute, ${ }^{3}$ Texas \\ A\&M Health Science Center, College \\ of Medicine, ${ }^{4}$ The Institute of \\ Metabolic Disease, ${ }^{5}$ Baylor University \\ Medical Center, Dallas, ${ }^{6}$ The Heart \\ Hospital Baylor, Plano, TX, USA
}

Introduction: Biomarkers reported as being less than the limit of detection (LOD) are challenging to be included in analyses without being dichotomized. Substitutions by the LOD, $\mathrm{LOD} / 2, \mathrm{LOD} / \sqrt{2}$, or zero have been attempted for left-censored values. We calculated a novel Modified Myocardial Injury Summary Score (MMISS) by incorporating four biomarkers (B-type natriuretic peptide, troponin, galectin-3, and suppression of tumorigenicity 2) to efficiently stratify heart failure (HF) patients. The objective of this study is to evaluate the differences in associations between MMISS, calculated by different methods of substitution, and therapeutic intensity index (TII), a composite pharmacologic score.

Methods and results: This is a cross-sectional study including $39 \mathrm{HF}$ patients aged $\geq 18$ years who were treated in the Baylor Health Care System. MMISS was calculated with the left-censored biomarkers substituted by zero, $\mathrm{LOD}, \mathrm{LOD} / 2$, and $\mathrm{LOD} / \sqrt{2}$. Patients with biomarker values more than LOD (complete cases) were also considered separately. The computed TII was regressed separately on MMISS for each substitution method, while controlling for age and gender. All substitution methods yielded negative associations; however, statistical significance for the association was not achieved using substitution by zero or when considering only the complete cases. The association was quite comparable with the substitution of left-censored values by LOD, LOD/2, and LOD/ $\sqrt{ } 2$. Conclusion: Substantial loss of information is inevitable if only the data with values above the LOD are considered for analysis or when the left-censored values are substituted with zero. Keywords: cardiac biomarkers, left-censored, limit of detection, therapeutic index, myocardial injury summary score

\section{Introduction}

Heart failure (HF) is associated with significant morbidity and mortality. According to the National Vital Statistics, age-adjusted HF mortality in the USA decreased from 2000 through 2012 but increased for the period of 2012-2014. ${ }^{1}$ HF is a complex condition where either the heart fails to pump blood adequately or has impaired ventricular filling. HF may be chronic when the symptoms appear slowly with progressive worsening of the condition or it may be acute most often following a heart attack or sudden inability to compensate the existing HF. Cardiac biomarkers are routinely used in the treatment of HF for diagnostic and prognostic purposes. Four biomarkers, namely troponin I or T, B-type natriuretic peptide or N-terminal (BNP), galectin-3, and suppression of tumorigenicity 2 (ST2), have been recommended by the American College of Cardiology Foundation (ACCF) and the American Heart Association (AHA) for effective management of $\mathrm{HF}^{2}$ We created a novel score incorporating these four biomarkers so as to efficiently stratify and therapeutically manage patients with HF. Quantification
Correspondence: Peter A McCullough Baylor Heart and Vascular Institute, 62I N. Hall Street, Suite H030, Dallas, TX 75226, USA

Tel + I 2484446905

Fax + I 2148207997

Email peteramccullough@gmail.com
Pathology and Laboratory Medicine International 2017:9 I5-20

\section{5}

(c) (i) (2) ( 2017 Vasuderan et al. This work is published and licensed by Dove Medical Press Limited. The full terms of this license are avalable at https://wwr.dovepress.com/terms.

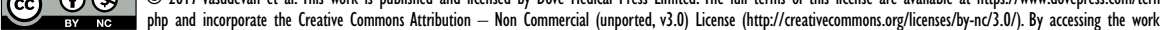
you hereby accept the Terms. Non-commercial uses of the work are permitted without any further permission from Dove Medical Press Limited, provided the work is properly attributed. For permission for commercial use of this work, please see paragraphs 4.2 and 5 of our Terms (httpss//www.dovepress.com/terms.php). 
of these biomarkers is dependent on the sensitivity of the diagnostic tool to detect the minimum reportable levels. In instances where the biomarkers are not detectable, they are simply reported as being less than the limit of detection (LOD) by the laboratory. Biomarkers reported as being less than the LOD are a challenge to be included in analyses without use of dichotomization, as their quantitative levels cannot be precisely defined. Substitution is performed in those instances for the biomarkers reported below the LOD, as they are essential for calculation of this prognostic score at the bedside. Traditional methods have advocated substitution with the LOD, LOD/2, LOD/ $\sqrt{ } 2$, or zero for the left-censored values. The objective of this study is to evaluate the differences in the association between therapeutic intensity index (TII) and the Modified Myocardial Injury Summary Score (MMISS), as calculated via different methods of substitution for the left-censored biomarkers.

\section{Methods}

This is a cross-sectional study including HF patients aged $\geq 18$ years who were treated at the outpatient clinics of the Baylor Health Care System. HF was defined as mentioned in the 2013 ACCF/AHA guidelines, which further categorized patients with ejection fraction $(\mathrm{EF}) \leq 40$ as $\mathrm{HF}$ with reduced EF (HFrEF). Values of the biomarkers were obtained at baseline and were used to calculate the MMISS, a novel score to better stratify HF patients. For biomarkers with values reported simply as "below the LOD", we imputed values using the $\mathrm{LOD}, \mathrm{LOD} / 2, \mathrm{LOD} / \sqrt{ } 2$, and zero to calculate the MMISS. Additionally, only those patients with biomarker values more than LOD were considered separately. The laboratory equipment was able to detect values as low as $0.02 \mathrm{ng} / \mathrm{mL}$ for troponin and $10 \mathrm{pg} / \mathrm{mL}$ for BNP.

\section{Calculation of MMISS}

The MMISS is calculated as an average of log-transformed ratios of a biomarker's present value to its previous value (obtained during the preceding visit). As this is a crosssectional study, subjects did not have biomarker values available from a preceding visit. Thus, the upper limit normal was used as a surrogate value for the prior clinical visit. That is

$$
\begin{aligned}
\text { MMISS } & =\frac{\sum_{i} \log _{10} \frac{\text { Biomarker }(i)_{\text {Measured }}}{\text { Biomarker }(i)_{\text {Baseline }}}}{n} \\
& =\frac{\sum_{i} \log _{10} \frac{\text { Biomarker }(i)_{\text {Measured }}}{\text { Biomarker }(i)_{\mathrm{ULN}}}}{n}
\end{aligned}
$$

where $i$ is the biomarker and $n$ is the total number of available biomarkers.

\section{Calculation of TII}

Based on the medications prescribed at the time of the biomarker draw, a TII was calculated. Based on the intensity of the therapy, scores were assigned for each medication and was found to range from 0 to 24 . The drug classes included for calculation of the TII were long-acting nitrates, beta-adrenergic blockers, angiotensin-converting enzyme inhibitors, angiotensin receptor blockers, mineralocorticoid receptor antagonists, hydralazine, digoxin, and loop diuretics.

\section{Statistical analysis}

The baseline characteristics of the patients included in the analyses were presented as means or medians for continuous variables and proportions for categorical variables. Comparisons were performed using the Wilcoxon rank-sum test or Student's $t$-test for continuous variables and the chi-square test or Fisher's exact test for proportions, where applicable.

Separate regression models were built using TII as the dependent variable and MMISS (as calculated via different substitution methods) as the independent variable, while controlling for age and gender. The objective was to identify any notable differences in the association of TII and MMISS, as obtained by the different substitution methods. The regression coefficient, standard error, and its statistical significance were compared for all substitution methods. Analyses were completed using STATA 14.1 and $p$ values $<0.05$ were considered statistically significant. Posthoc power calculation was conducted using SAS 9.4 (Cary, NC, USA). With a sample size of 39 patients, we had $\sim 88 \%$ power to detect a partial correlation of -0.48 between MMISS and TII while controlling for age and gender. This study was approved by the Baylor Institutional Review Board with a waiver of consent as this study involved an anonymous review of existing medical information posing minimal risk to the subjects.

\section{Results}

There were 39 patients included in this study, and their characteristics are in Table 1. According to the New York Heart Association (NYHA) functional classification, 13 (33.3\%) patients had class II symptoms, 22 (56.4\%) had class III symptoms, and the remaining $4(10.3 \%)$ patients had class IV symptoms. The mean left ventricular ejection fraction was $40.6 \pm 17.6 ; 48.7 \%$ with HFrEF. The components of TII and the frequency of the drugs prescribed in each of the 
categories are tabulated in Table 2 . There were $4(10.3 \%)$ and $29(74.4 \%)$ instances with left-censored values for BNP and troponin, respectively. Only 9 (23.1\%) patients had all biomarker values noted above the LOD and were included in the complete case analysis.

MMISS calculated for the complete cases and by different substitution methods for left-censored biomarkers (LOD, $\mathrm{LOD} / 2, \mathrm{LOD} / \sqrt{ } 2$, zero) are represented in Table 3 and graphically by gender (Figure 1) and NYHA functional classification (Figure 2). The regression parameter estimates for MMISS under different substitution methods are in Table 4. Each method revealed a negative association between MMISS and TII in univariate and multivariate analyses. In the multivariate analyses, the regression coefficients of MMISS obtained by including the complete data (patients with available biomarker values more than $\mathrm{LOD} ; p=0.28$ ) and also by substituting zeroes

Table I Characteristics of patients included in the study $(n=39)$

\begin{tabular}{ll}
\hline Variables & Value \\
\hline Median age (years; range) & $60(39-84)$ \\
Median BMI (kg/m² range) & $29.8(19.5-47.2)$ \\
Male, $\mathrm{n}(\%)$ & $21(53.8)$ \\
Ischemic cardiomyopathy, $\mathrm{n}(\%)$ & $20(51.3)$ \\
Diabetes mellitus, $\mathrm{n}(\%)$ & $23(58.9)$ \\
Hypertension, $\mathrm{n}(\%)$ & $34(87.1)$ \\
Dyslipidemia, $\mathrm{n}(\%)$ & $33(84.6)$ \\
Peripheral vascular disease, $\mathrm{n}(\%)$ & $6(15.4)$ \\
Chronic kidney disease, $\mathrm{n}(\%)$ & $1 \mathrm{I}(28.2)$ \\
Atrial fibrillation, $\mathrm{n}$ (\%) & $10(25.6)$ \\
Prior myocardial infarction, $\mathrm{n}(\%)$ & $22(56.4)$ \\
Number of left-censored values, BNP, $\mathrm{n}(\%)$ & $4(10.3)$ \\
Number of left-censored values, troponin, $\mathrm{n}(\%)$ & $30(76.9)$ \\
\hline
\end{tabular}

Abbreviations: BMI, body mass index; BNP, B-type natriuretic peptide or N-terminal.

Table 2 Components of therapeutic intensity index with frequency of the drugs prescribed

\begin{tabular}{ll}
\hline Therapy & Patients (n, \%) \\
\hline ACEI & $14(35.9)$ \\
ARB & $8(20.5)$ \\
Beta-blocker & $29(74.4)$ \\
MRA & $10(25.6)$ \\
Long acting nitrates & $5(12.8)$ \\
Hydralazine & $6(15.4)$ \\
Digoxin & $6(15.4)$ \\
Loop diuretic & $21(53.8)$ \\
\hline
\end{tabular}

Abbreviations: $A C E l$, angiotensin-converting enzyme inhibitor; $A R B$, angiotensin receptor blockers; MRA, mineralocorticoid receptor antagonists. for the left-censored values were not statistically significant ( $p=0.05$ ) with an adjusted $R^{2}$ of 0.07 . The regression coefficients of MMISS obtained by substituting the left-censored biomarkers with $\operatorname{LOD}(p=0.02), \mathrm{LOD} / 2(p=0.01)$, and LOD $/ \sqrt{ } 2$ $(p=0.02)$ were statistically significant with an adjusted $R^{2}$ of $0.11,0.13$, and 0.12 , respectively.

\section{Discussion}

The association between TII and MMISS varied with the different methods of substitution for the left-censored biomarkers. Although all the methods yielded a negative relation, the association did not reach statistical significance when substituted by zero or when only cases with values more than LOD were included. The association was quite comparable with the substitution of left-censored values by LOD, LOD/2, and $\mathrm{LOD} / \sqrt{2}$. We found important relationships between MMISS and NYHA as observed in Figure 2, demonstrating that all four imputation methods had graded increases in MMISS by NYHA classification, suggesting biologic responsiveness to the severity of disease.

Analysis of left-censored biomarker data need special attention as different methods may lead to different conclusions. Often, censored biomarkers are dichotomized so as to explore their relation with the clinical outcomes. ${ }^{3}$ In situations where $>10 \%$ of the values are left-censored, it has been suggested to use multiple imputation techniques for effective estimates. Tobit analysis has also been recommended to statistically model left-censored detections for robust measurements of association. ${ }^{4}$ We employed only the traditional substitution methods as our intention was to incorporate the biomarkers in a prognostic clinical score for effective management of patients with HF. It has been suggested that these traditional methods of substitution can be used, provided they do not compromise the validity of the study. ${ }^{5}$ Ignoring those values noted as below LOD or substituting them with a zero introduces unnecessary bias resulting in erroneous assumptions. In a clinical setting, values below the LOD may be useful in establishing a diagnosis but would not be able to offer details for further stratification of the severity of the disease.

In 2013, the ACCF and the AHA suggested that four blood biomarkers could be useful in advanced risk strati-

Table 3 Biomarker values and MMISS by different substitution methods (median [range])

\begin{tabular}{|c|c|c|c|c|c|}
\hline & LOD $(n=39)$ & LOD/2 (n=39) & $\operatorname{LOD} / \sqrt{ } 2(n=39)$ & Zero $(n=39)$ & Complete cases $(n=9)$ \\
\hline BNP & 107 (10-2440) & $107(5-2440)$ & 97.75 (7.07-2440) & $107(0-2440)$ & 419 (80-969) \\
\hline Troponin & $0.02(0.02-I .7 I)$ & $0.0 \mathrm{I}(0.0 \mathrm{I}-\mathrm{I} .7 \mathrm{I})$ & $0.014(0.014-1.71)$ & $0(0-1.7 I)$ & $0.04(0.02-I .7 I)$ \\
\hline MMISS & $-0.089(-0.61-1.08)$ & $-0.189(-0.76-1.08)$ & $-0.166(-0.69-1.08)$ & $0.049(-0.41-1.08)$ & $0.335(-0.097-1.08)$ \\
\hline
\end{tabular}

Abbreviations: BNP, B-type natriuretic peptide or N-terminal; LOD, limit of detection; MMIS, Modified Myocardial Injury Summary Score. 


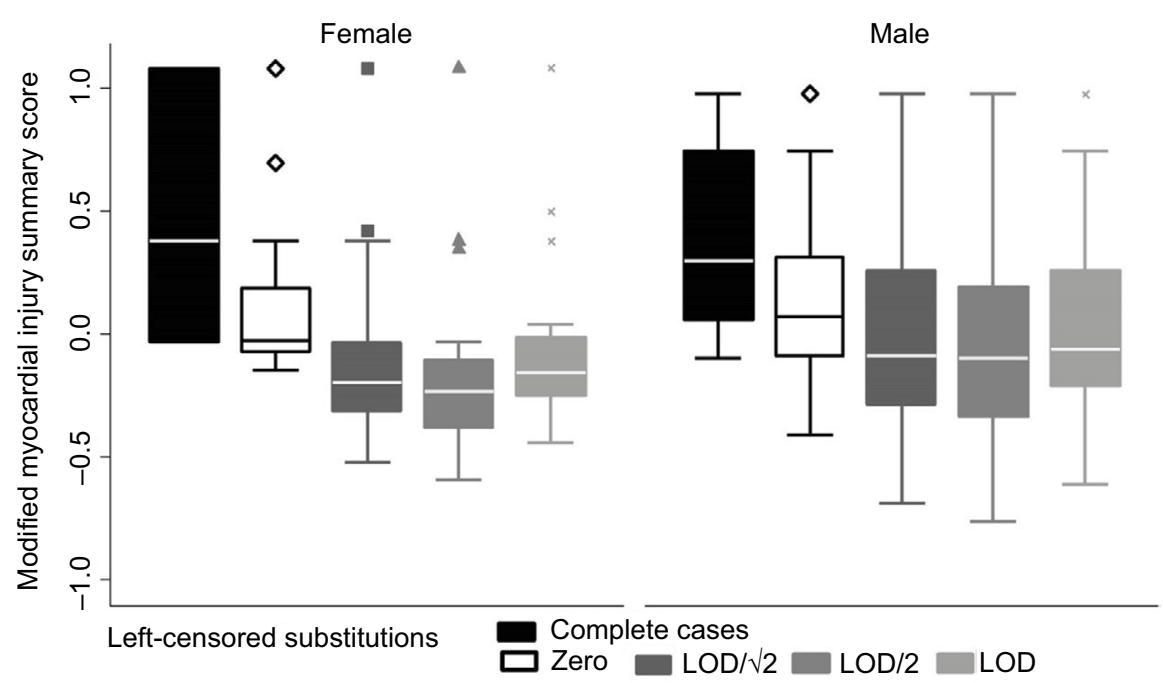

Figure I Distribution of modified myocardial injury by different methods of substitution of left-censored biomarkers by gender. Abbreviation: LOD, limit of detection.

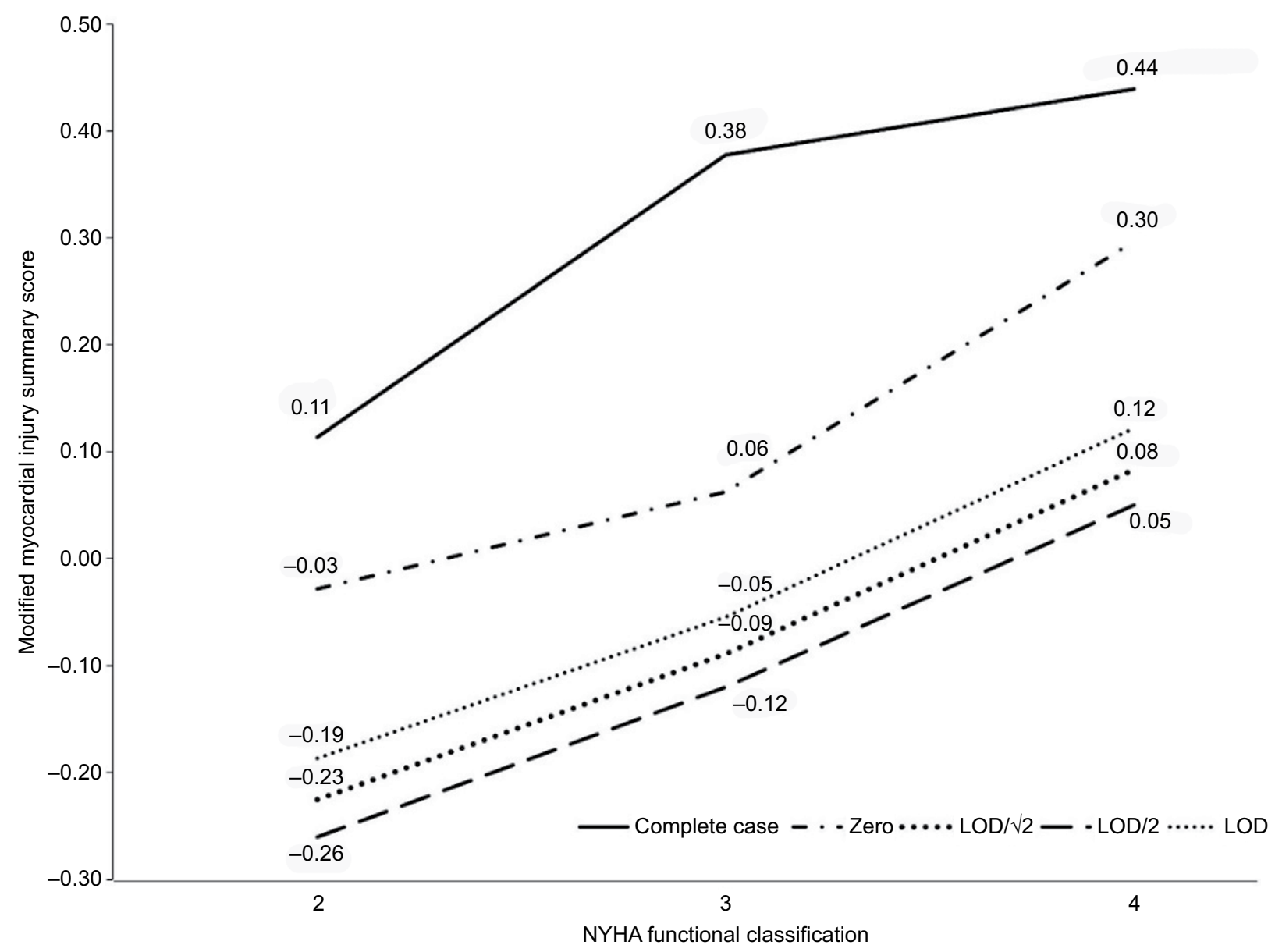

Figure 2 Median values of modified myocardial injury by different methods of substitution of left-censored biomarkers by NYHA functional class. Abbreviations: NYHA, New York Heart Association; LOD, limit of detection.

fication and guideline-directed medical therapy in patients with chronic $\mathrm{HF}^{2}$ These markers include troponin I or T, BNP or N-terminal BNP, galectin-3, and ST2. Trials have shown BNP or NT-pro BNP guided therapy to be associated with reduced mortality ${ }^{6}$ but none of the previous trials have incorporated troponin I or T, galectin-3, or ST2. ${ }^{7}$ ST2 has been recommended as prognostic markers in patients suffering from myocardial infarction, HF, and dyspnea. ${ }^{8}$ Studies 
have also associated ST2 with plaque development and rupture of atheromas. ${ }^{9}$ We incorporated the four biomarkers in a prognostic tool that can be used at the bedside for effective clinical management.

The study has all the potential limitations of a crosssectional study with a limited sample size. No causal inference can be made as the data were collected at a single point in time. As the true regression coefficient is unknown, we are unsure of the acceptable substitution methodology. To further study the performance of the different substitution methods (LOD, $\mathrm{LOD} / 2$, and $\mathrm{LOD} / \sqrt{2}$ ), we would need a larger sample size with biomarker values collected sequentially over time.

\section{Conclusion}

Substantial loss of information becomes inevitable if only the data with values above LOD are considered for analysis or when the left-censored values are substituted with a zero. There are several substitution methods for left censoring that yield similar results. The MMISS is not associated with the intensity of medications prescribed for HF, but does appear to be quantitatively related to the physician-assigned severity of illness.

\section{Disclosure}

The authors report no conflicts of interest in this work.

\section{References}

1. Ni H, Xu JQ. Recent Trends in Heart Failure-Related Mortality: United States, 2000-2014. NCHS data brief, no 231. Hyattsville, MD: National Center for Health Statistics. 201.

2. Yancy CW, Jessup M, Bozkurt B, et al; American College of Cardiology Foundation; American Heart Association Task Force on Practice Guidelines. $2013 \mathrm{ACCF} / \mathrm{AHA}$ guideline for the management of heart failure: a report of the American College of Cardiology Foundation/American Heart Association Task Force on Practice Guidelines. J Am Coll Cardiol. 2013 Oct 15;62(16):e147-e239.

3. Whitcomb BW, Schisterman EF. Assays with lower detection limits: implications for epidemiological investigations. Paediatr Perinat Epidemiol. 2008;22(6):597-602.

4. Lubin JH, Colt JS, Camann D, et al. Epidemiologic evaluation of measurement data in the presence of detection limits. Environ Health Perspect. 2004;112(17):1691-1696.

5. Ogden TL. Editor's choice: handling results below the level of detection. Ann Occup Hyg. 2010;54(3):255-256.

6. Troughton RW, Frampton CM, La Rocca HPB, et al. Effect of B-type natriuretic peptide-guided treatment of chronic heart failure on total mortality and hospitalization: an individual patient meta-analysis. Eur Heart J. 2014;35(23):1559-1567.

7. McCullough PA. Practical experience using galectin-3 in heart failure. Clin Chem Lab Med. 2014;52(10):1425-1431.

8. Ciccone MM, Cortese F, Gesualdo M, et al. A novel cardiac bio-marker: ST2: a review. Molecules. 2013;18(12):15314-15328.

9. Marzullo A, Ambrosi F, Inchingolo M, et al. ST2L transmembrane receptor expression: an immunochemical study on endarterectomy samples. PLoS One. 11(5):e0156315. 


\section{Publish your work in this journal}

Pathology and Laboratory Medicine International is a peer-reviewed, open access journal focusing on innovative basic research and translational research related to pathology or human disease. The journal includes original research, updates, case reports, reviews and commentaries on current controversies. The manuscript management system is completely online and includes a very quick and fair peer-review system. Visit http://www.dovepress.com/testimonials.php to read real quotes from published authors.

Submit your manuscript here: https://www.dovepress.com/pathology-and-laboratory-medicine-international-journal 